\title{
EP-39
}

\section{Caudate lobe abscess: Challenging site with less invasive option of treatment: A case series}

\author{
Farah Najla SUPANDI ${ }^{* 1,4}$, Balraj SINGH', Rizal ROSLAN², Izazul HUSIN², Tiong See LEE ${ }^{3}$ \\ 'Department of HPB Surgery and Liver Transplantation, Hospital Selayang, Selangor, Malaysia \\ ${ }^{2}$ Department of Interventional Radiology, Hospital Selayang, Selangor, Malaysia \\ ${ }^{3}$ Department of Gastroenterology and Hepatology, Hospital Selayang, Selangor, Malaysia \\ ${ }^{4}$ Department of Surgery, Faculty of Medicine, Universiti Teknologi MARA, Selangor, Malaysia
}

Introduction: The rarity of caudate lobe abscess has made it difficult to outline a treatment algorithm for this pathology due to its anatomically challenging site. Caudate lobe abscesses also poses a risk of rupture, making its management more complicated. However, advances in imaging have made it possible for early detection and paradigm shift in its management from open surgery to non-operative intervention.

Methods: All patients with isolated caudate lobe abscess were selected retrospectively and diagnosis was confirmed by imaging. Patient in this case series underwent either percutaneous or endoscopic drainage and empirical antibiotic therapy alone.

Results: There are 5 patients in this case series, 3 of them underwent percutaneous drainage, 1 of them treated with antibiotic alone and another underwent endoscopic ultrasound (EUS)-guided drainage. All patients showed complete resolution of caudate lobe abscess without procedural complications.

Conclusions: The caudate lobe, which lies posterior to the hepatoduodenal ligament and anterior to the inferior vena cava exposes the patient to possible life-threatening complications when subjected to drainage via a percutaneous approach. EUS allows not only identification of intervening vessels which is the major reason for technical difficulty in percutaneous drainage, but excellent visualization of abscess cavities and the surrounding landmarks. In conclusion, non-surgical approach is our treatment of choice in management of caudate lobe liver abscess. 